\title{
A Neural Network Tool for the Interpolation of foF2 Data in the Presence of Sporadic E Layer
}

\author{
Haris Haralambous, Antonis Ioannou, and Harris Papadopoulos \\ Computer Science and Engineering Department, Frederick University, \\ 7 Y. Frederickou St., Palouriotisa, Nicosia 1036, Cyprus \\ $\{$ H. Haralambous, H. Papadopoulos\} @efrederick . ac . cy
}

\begin{abstract}
This paper presents the application of Neural Networks for the interpolation of (critical frequency) foF 2 data over Cyprus in the presence of sporadic $\mathrm{E}$ layer which is a frequent phenomenon during summer months causing inevitable gaps in the $f o F 2$ data series. This ionospheric characteristic ( $f o F 2$ ) constitutes the most important parameter in HF (High Frequency) communications since it is used to derive the optimum operating frequency in $\mathrm{HF}$ links and therefore interpolating missing data is very important in preserving the data series which is used in long-term prediction procedures and models.
\end{abstract}

Keywords: Ionosphere, HF communications, F2 layer critical frequency.

\section{Introduction}

The ionosphere is defined as a region of the earth's upper atmosphere where sufficient ionisation can exist to reflect radio waves in the frequency range 1 to $30 \mathrm{MHz}$ therefore facilitating long distance HF (High Frequency) communication by skywave propagation. It ranges in height above the surface of the earth from approximately 50 $\mathrm{km}$ to $1000 \mathrm{~km}$. The influence of this region on radio waves is accredited to the presence of free electrons.

The uppermost layer of the ionosphere is the F2 region which is the principal reflecting region for long distance $\mathrm{HF}$ communications [1,2,3]. The maximum frequency that can be reflected at vertical incidence by this layer is termed the F2 layer critical frequency ( $f o F 2$ ) and is directly related to the maximum electron density of the layer (see figure 1). The F2 layer critical frequency is the most important parameter in HF communication links since when multiplied by a factor which is a function of the link distance, it defines the optimum usable frequency of operation. The maximum electron density of free electrons within the F2 layer and therefore foF 2 depend upon the strength of the solar ionising radiation which is a function of time of day, season, geographical location and solar activity $[1,2,3]$. The E-region lies below the F-region extending approximately from $90 \mathrm{~km}$ to $150 \mathrm{~km}$ (figure 1). Typically the E-region is characterised by lower electron densities than the F-region (continuous profile in figure 1). However sometimes an irregular layer of extremely high density layer called sporadic E (Es) layer appears (dashed part in the profile in figure 1) in the E-region which prevents any radio signals from reaching the F-region. Es can occur during daytime or night-time, and its characteristics vary with latitude. 
These regions can apparently be caused by several mechanisms, and have a wide variety of characteristics. They have been correlated in literature with solar activity, geomagnetic activity and associated with thunderstorms and meteor showers [4,5].

This paper describes the development of a neural network tool to interpolate missing foF 2 data over Cyprus. The tool development is based on 10342 hourly foF 2 measurements recorded above Cyprus from September 2008 to August 2010. The practical application of this model lies in the fact that in the presence of Es above Cyprus, due to the inability of the ionosonde to perform foF 2 measurements, this model can provide an efficient interpolation method to fill the gaps in the foF 2 data series that is used to drive long-term prediction models of $f o F 2$. There have not been any other efforts to apply interpolation techniques on foF2 datasets apart from a specific dataset over UK [6]. This can be partly explained by the fact that most ionospheric stations are situated at middle latitudes where Es is not a frequent phenomenon and so data gaps may only appear because of system failure. In the case of Cyprus which is the lowest European ionospheric station in operation, the Es phenomenon is very frequent causing data gaps under normal system operation. A possible candidate for the interpolation procedure would have been the recently developed neural network approach from modelling foF2 [7]. However the key novelty of the proposed interpolation tool is the use of TEC measurements as an additional input resulting in improved performance compared to the existing longterm (without TEC as input) model in terms of interpolation.

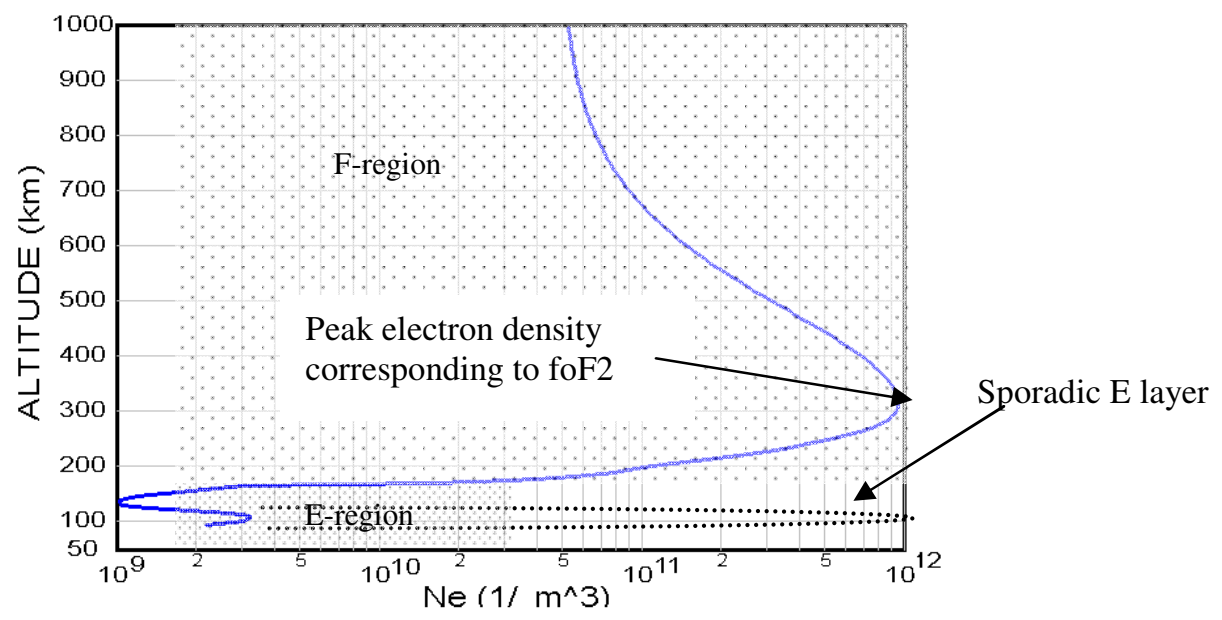

Fig. 1. Typical electron density altitude profile of the ionosphere superimposed with enhanced density in the E-region due to Es layer

\section{Measurement of F2 Layer Critical Frequency and Total Electron Content}

Measurements of foF 2 are conducted by ionosondes. which sweep through the HF frequency range transmitting short pulses. These pulses are reflected at various layers 
of the ionosphere, and their echoes are received by the receiver giving rise to a corresponding plot of reflection altitude against frequency (called an ionogram- figure 2) which is further analysed to infer the ionospheric plasma height-electron density profile (figure 1). The maximum frequency at which an echo is received is called the critical frequency of the corresponding layer. Since the F2 layer is the most highly ionised ionosperic layer its critical frequency foF 2 is the highest frequency that can be reflected by the ionosphere (figure $2 b$ ). The presence of a Es layer (figure 2a) which does not allow for ionosonde signals to reach F2 region altitudes does not allow a useful ionogram to be obtained and therefore gaps in the data series of foF 2 are formed (figure 3b). These gaps have to be interpolated in a way to preserve the inherent variability of $f o F 2$ data. This study suggests that one way to achieve this is to incorporate Total Electron Content (TEC) measurements which is another type of ionospheric characteristic the measurement of which is not affected by Es layer. The reason is that the signals involved in the measurement of TEC are high enough $(>1$ $\mathrm{GHz}$ ) to pass through the Es layer practically unaffected as opposed to the much lower frequency of ionosonde signals $(<20 \mathrm{MHz})$.

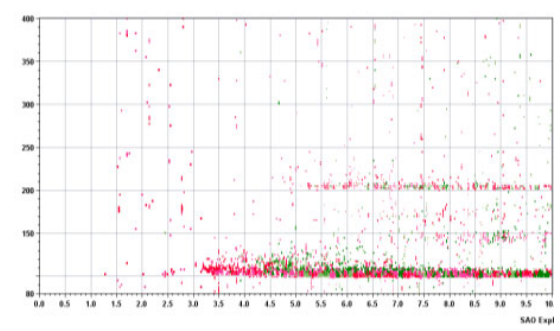

(a)

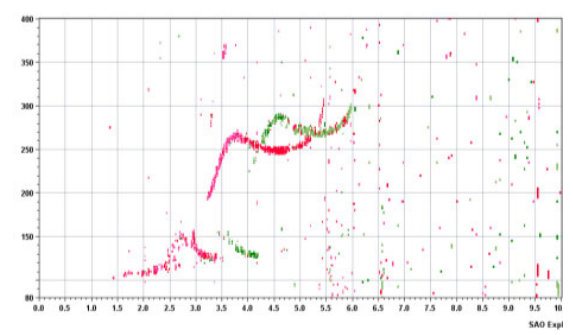

(b)

Fig. 2. Ionogram (a) with Es blocking F2 layer reflections (b) with normal F2 reflections in the absence of Es layer

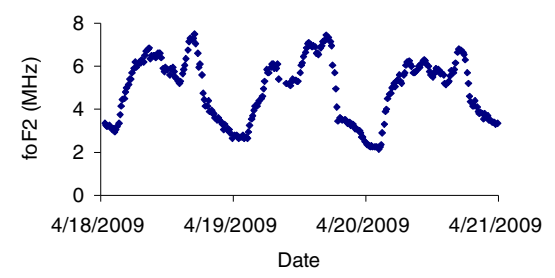

(a)

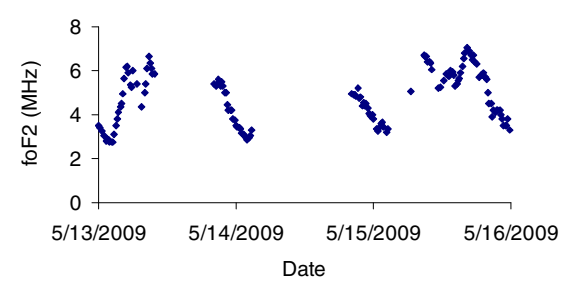

(b)

Fig. 3. Data series of foF2 (a) without gaps (b) with gaps due to ionograms with Es layer

Dual-frequency GPS data recorded by GPS (Global Positioning System) receivers enable an estimation of TEC because of the frequency dependent delay imposed on the signal due to the ionosphere [8]. By processing code and phase measurements on two frequencies in the L-band ( $\mathrm{L} 1=1575.42 \mathrm{MHz}, \mathrm{L} 2=1227.60 \mathrm{MHz}$ ) it is possible to 
extract an estimate of the TEC measured in total electron content units, $(1$ TECU = $\left.10^{16} \mathrm{el} \mathrm{m}-2\right)$. This is the total amount of electrons along a particular line of sight between the receiver and a GPS satellite in a column of $1 \mathrm{~m}^{2}$ cross-sectional area. TEC is therefore the integral of the electron density profile (figure 1) from the Eregion up to the height of the GPS satellite encompassing the electrons in the F-region as well.

The analysis used in the present work to estimate TEC from GPS transmissions was carried out by means of the procedure developed by Ciraolo [9,10]. It can be shown that the measurable GPS ionospheric delay is expressed in terms of TEC as [11]:

$$
t_{\text {ion }}=\frac{40.3}{c f^{2}} T E C
$$

and

$$
T E C=\int_{h 1}^{h 2} N(h) \cdot d h
$$

$\mathrm{t}_{\text {ion }}$... GPS ionospheric delay

c... velocity of light in free space

f... frequency of radio wave propagating through the ionosphere (GPS radio signal)

$\mathrm{N}(\mathrm{h})$... ionospheric electron density at the height h above the earth's surface

TEC ... total electron content along the satellite ray path.

\section{Characteristics of the F2 Layer Critical Frequency and Total Electron Content}

Solar activity has an impact on ionospheric dynamics which in turn influence the electron density of the ionosphere. The electron density of the F2 layer and TEC exhibit variability on daily, seasonal and long-term time scales in response to the effect of solar radiation. It is also subject to abrupt variations due to enhancements of geomagnetic activity following extreme manifestations of solar activity disturbing the ionosphere from minutes to days on a local or global scale.

The most profound solar effect on foF 2 and TEC is reflected on their daily variation as shown in figure 4 . As it is clearly depicted, there is a strong dependency on local time which follows a sharp increase of foF 2 and TEC around sunrise and gradual decrease around sunset. This is attributed to the rapid increase in the production of electrons due to the photo-ionization process during the day and a more gradual decrease due to the recombination of ions and electrons during the night. The long-term effect of solar activity on both parameters follows an eleven-year cycle and is clearly shown in figure 5(a),(b) where all values of $f o F 2$ and TEC are plotted against time as well as a modeled monthly mean sunspot number $R$ which is a well established index of solar activity. We can observe a marked correlation of the mean level of both parameters and modeled sunspot number. In addition to the effects of 
solar activity on both parameters mentioned above we can also identify a strong effect on the diurnal variability as solar activity gradually increases through its 11-year cycle. This is demonstrated again in figure 4 where the diurnal variation of foF 2 and TEC is plotted for three different days corresponding to low, medium and high sunspot number periods. It is evident from this figure that the night to day variability is increased as sunspot number increases for both parameters.

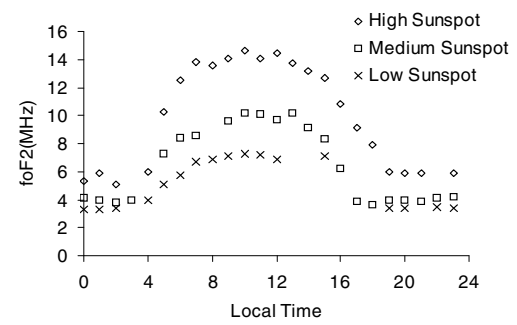

(a)

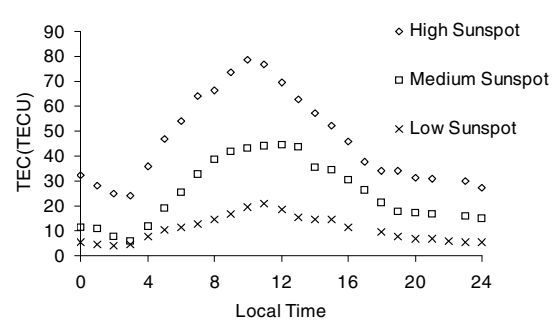

(b)

Fig. 4. Diurnal variability of (a) foF2 and (b)TEC for low, medium and high solar activity
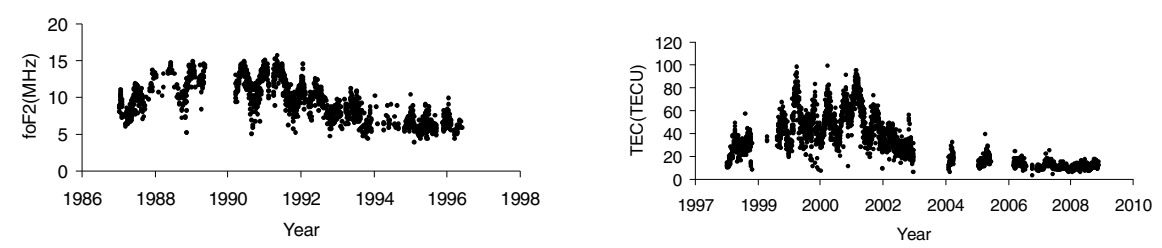

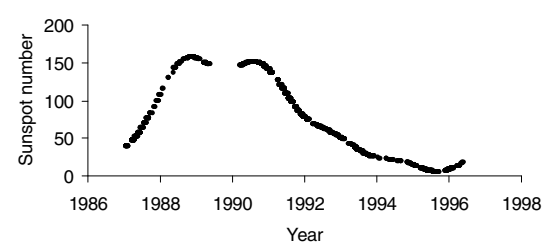

(a)

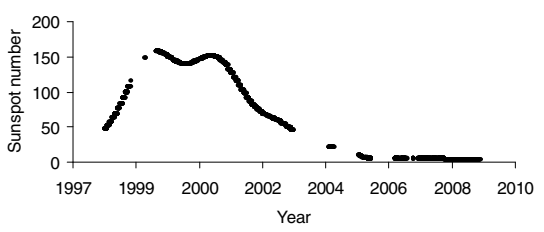

(b)

Fig. 5. Long-term variability of (a) foF 2 and (b)TEC and solar activity

There is also a seasonal component in the variability of foF2 and TEC which can be attributed to the seasonal change in extreme ultraviolet (EUV) radiation from the Sun. This can be clearly identified in figure 6 for noon values of both parameters for two different years.

From the evidence drawn from this section the incorporation of TEC as an input to the neural network interpolation tool for $f o F 2$ data is justified based on its established correlation with TEC. However as shown in other studies this correlation varies diurnally and seasonally and also it is established that foF2 is not linearly related to TEC [12]. 


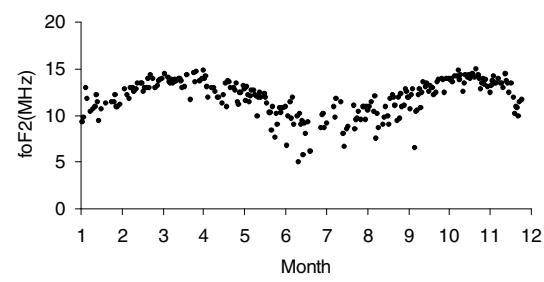

(a)

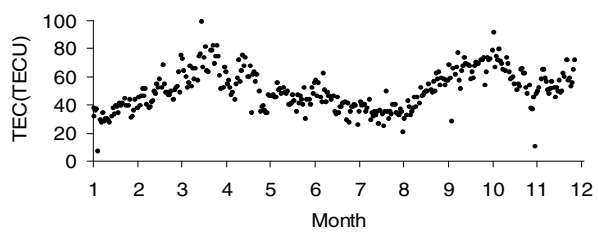

(b)

Fig. 6. Seasonal variability of (a) foF2 and (b) TEC

\section{Model Parameters}

The diurnal variation of $f o F 2$ is clearly evident by observing figure 4 . We therefore include hour number as an input to the model. The hour number, hour, is an integer in the range $0 \leq$ hour $\leq 23$. In order to avoid unrealistic discontinuity at the midnight boundary, hour is converted into its quadrature components according to:

$$
\text { sinhour }=\sin \left(2 \pi \frac{\text { hour }}{24}\right)
$$

and

$$
\text { coshour }=\cos \left(2 \pi \frac{\text { hour }}{24}\right)
$$

A seasonal variation is also an underlying characteristic of foF 2 as shown in figure 6 and is described by day number daynum in the range $1 \leq$ daynum $\leq 365$. Again to avoid unrealistic discontinuity between December 31st and January 1st daynum is converted into its quadrature components according to:

$$
\text { sinday }=\sin \left(2 \pi \frac{\text { daynum }}{365}\right)
$$

and

$$
\operatorname{cosday}=\cos \left(2 \pi \frac{\text { daynum }}{365}\right)
$$

Long-term solar activity has a prominent effect on foF 2. To include this effect in the model specification we need to incorporate an index, which represents a good indicator of solar activity. In ionospheric work the 12-month smoothed sunspot number is usually used, yet this has the disadvantage that the most recent value available corresponds to $f o F 2$ measurements made six months ago. To enable foF 2 data to be modelled as soon as they are measured, and for future predictions of $f o F 2$ to be made, the monthly mean sunspot number values were modeled using a smooth 
curve defined by a summation of sinusoids (figure 5). TEC is the last parameter used as an input to the neural network tool. It corresponds to the value of TEC measured simultaneously as the foF2 value. So the tool makes use of the available TEC values to generate the missing values of foF 2 when the Es layer is present.

\section{Experiments and Results}

To test the performance of this approach in interpolating missing foF 2 values we followed a 10-fold cross-validation process; the 10342 foF 2 values (recorded above Cyprus from September 2008 to August 2010) and their corresponding inputs were split into 10 continuous parts of almost equal size and the predictions for each part were obtained by training a neural network on the other 9 parts. We chose to split the data into blocks of continuous values in order to test the performance of the approach in a worst-case scenario where the missing values cover a relatively big period of time.

The neural networks used were fully connected two-layer networks, with 6 input, 5 hidden and 1 output neuron. Both its hidden and output neurons had hyperbolic tangent sigmoid activation functions. The number of hidden neurons was determined by trial and error. The training algorithm used was the Levenberg-Marquardt backpropagation algorithm with early stopping based on a validation set created from $20 \%$ of the training examples. In an effort to avoid local minima ten neural networks were trained on each training set with different random initializations and the one that performed best on the corresponding validation set was selected for application to the test examples. The inputs and target outputs of the network were normalized setting their minimum value to -1 and their maximum value to 1 . This made the impact
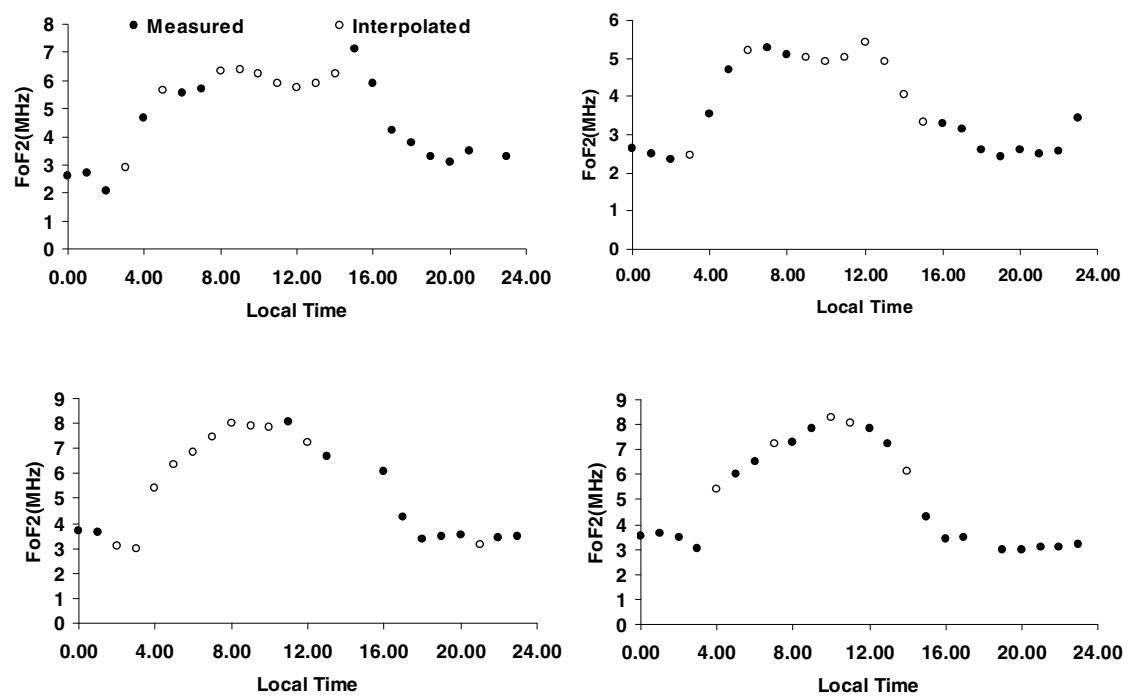

Fig. 7. Examples of measured and interpolated foF2 values 
of all inputs in the model equal and transformed the target outputs to the output range of the neural network activation functions. The results reported here were obtained by mapping the outputs of the network for the test examples back to their original scale. The whole process was implemented in Matlab with the aid of the Matlab Neural Network toolbox.

The obtained Root Mean Squared Error (RMSE) over all data was $0.508 \mathrm{MHz}$ and the Mean Absolute Error (MAE) was $0.383 \mathrm{MHz}$. In order to see the improvement resulting from the inclusion of TEC in the inputs of the neural network (i.e. by using the long-term prediction approach of [7]) we performed exactly the same experiment without including TEC. In this case the resulting RMSE was $0.712 \mathrm{MHz}$ and the resulting MAE was $0.545 \mathrm{MHz}$. This shows that by using TEC we achieve a much more accurate interpolation of missing foF 2 values. Finally examples of the approach are demonstrated graphically in figure 7.

\section{Conclusions and Future Work}

In this paper we have presented the development of a neural network tool for the interpolation of missing critical frequency data of the F2 ionospheric layer (foF2) above Cyprus due to sporadic E layer conditions. The model has been developed based on a data set obtained during a period of approximately two years. The tool has produced a good interpolation capability of the gaps in the foF 2 data series therefore providing a method to preserve the variability of $f o F 2$, a fact which is essential in the development of long-term time-series prediction models and procedures. The tool will be further evaluated as we collect more foF 2 measurements in the next few years.

\section{References}

1. Goodman, J.: HF Communications, Science and Technology. Nostrand Reinhold, New York (1992)

2. Maslin, N.: The HF Communications, a Systems Approach, San Francisco (1987)

3. McNamara, L.F.: Grid The Ionosphere: Communications, Surveillance, and Direction Finding. Krieger Publishing Company, Malabar (1991)

4. Mathews, J.D., Sporadic, E.: current views and recent progress. Journal of Atmospheric and Solar-Terrestrial Physics 60, 413-435 (1998)

5. Whitehead, J.D.: Recent work on mid-latitude and equatorial sporadic-E. Journal of Atmospheric and Terrestrial Physics 51, 401-424 (1989)

6. Francis, N.M., Brown, A.G., Cannon, P.S., Broomhead, D.S.: Nonlinear Prediction of the Hourly foF2 Times Series in Coonjunction with the Interpolation of Missing Data Points. Phys. Chem. Earth. 25(4), 261-265 (2000)

7. Haralambous, H., Papadopoulos, H.: A Neural Network model for the critical frequency of the F2 ionospheric layer over Cyprus. In: Palmer-Brown, D., Draganova, C., Pimenidis, E., Mouratidis, H. (eds.) EANN 2009. CCIS, vol. 43, pp. 371-377. Springer, Heidelberg (2009)

8. Wild, U., Beutler, G., Gurtner, W., Rothacher, M.: Estimating the Ionosphere Using One or More Dual Frequency GPS Receivers. In: Proceedings of the Fifth International Geodetic Symposium on Satellite Positioning, Las Cruces, New Mexico, March 13-17, vol. 2, pp. 724-736 (1989) 
9. Ciraolo L.: Evaluation of GPS L2-L1 biases and related daily TEC profiles. In: Proceedings of the GPS/Ionosphere Workshop, Neustrelitz, pp. 90-97 (1993)

10. Ciraolo, L.: Results and problems in GPS TEC evaluation. In: Proceedings of the Radio Communications Research Units First GPS TEC Workshop, Chilton, pp. 47-60 (2000)

11. Davis, K.: Ionospheric Radio. Peter Peregrinus Ltd., London (1990)

12. Spalla, P., Ciraolo, L.: TEC and foF2 comparison. Ann. Geofis. XXXVII (5), 929-938 (1994) 